\title{
Turing patterns in a predator-prey model with seasonality
}

\author{
Xiaoying Wang \\ Department of Mathematics and Statistics, University of Ottawa \\ Ottawa, ON K1N 6N5, Canada \\ Frithjof Lutscher \\ Department of Mathematics and Statistics, and Department of Biology, University of Ottawa \\ Ottawa, ON K1N 6N5, Canada
}

\begin{abstract}
Many ecological systems show striking non-homogeneous population distributions. Diffusion-driven instabilities are commonly studied as mechanisms of pattern formation in many fields of biology but only rarely in ecology, in part because some of the conditions seem quite restrictive for ecological systems. Seasonal variation is ubiquitous in temperate ecosystems, yet its effect on pattern formation has not yet been explored. We formulate and analyze an impulsive reaction-diffusion system for a resource and its consumer in a two-season environment. While the resource grows throughout the 'summer' season, the consumer reproduces only once per year. We derive conditions for diffusion-driven instability in the system, and we show that pattern formation is possible with a Beddington-DeAngelis functional response. More importantly, we find that a low overwinter survival probability for the resource enhances the propensity for pattern formation: diffusion-driven instability occurs even when the diffusion rates of prey and predator are comparable (although not when they are equal).
\end{abstract}

Keywords: Consumer-resource, impulsive reproduction, Turing bifurcation, pattern formation, reaction-diffusion equation, discrete system.

Mathematics Subject Classification: 35R12, 35B36, 92D25

\section{Introduction}

A central challenge in spatial ecology is to study the mechanisms generating the spatial distribution of organisms observed in the environment. This distribution may arise from and reflect the underlying distribution of abiotic conditions such as climatic, geophysical, or hydrological factors. However, as shown by Rietkerk and Van de Koppel (2008), non-homogeneous distributions of organisms are well documented even in homogeneous landscapes. The classical explanation for spatial patterns in a homogeneous landscape stems from Turing's work on diffusion-driven pattern formation between two interacting species (Turing 1952). Turing's original work related 
to morphogenesis and developmental patterns and requires two fundamental properties of the system: (i) an activator-inhibitor interaction structure of at least two interacting species, and (ii) an order of magnitude difference in their respective dispersal scales. An excellent explanation and review of applications of Turing's ideas can be found in the book by Murray (2001).

Turing's theory was used to explain patchy distributions of plankton (Levin and Segel 1976; Steele 1978), but it was not widely applied in ecology after the realization that the most commonly used predator-prey system, the MacArthur-Rosenzweig model, did not show the required activator-inhibitor structure. Additional nonlinear mechanisms are required, such as an Allee effect and self-limitation of the predator to produce diffusion-driven patterns in predator-prey systems (Mimura and Murray 1978; Morozov et al. 2006; Petrovskii et al. 2002; Wang et al. 2011). Alternatively, different functional forms, such as the Leslie-May model (Leslie and Gower 1960; May 2001) as well as several ecologically realistic extensions of the classical MacArthurRosenzweig model (Fasani and Rinaldi 2011; Shi and Ruan 2015) all give the required activatorinhibitor structure. In all these cases, the magnitude difference in dispersal scales is still a requirement for pattern formation. Such a difference is not easy to document empirically. Recent work by Cobbold et al. (2015) shows that spatial heterogeneity might alleviate the strict requirement of large dispersal differences. In the current work, we study to what extent temporal heterogeneity might weaken the two fundamental conditions for pattern formation.

Many ecosystems experience temporal heterogeneity in the form of seasonal variation of climatic conditions. Dispersal patterns and species interaction could depend on season. In addition, while some species grow and/or reproduce more or less continuously during at least one season (e.g. plants, several rodents), others reproduce only once during a year (e.g. many birds and mammals). The classical reaction-diffusion framework for Turing patterns assumes continuously reproducing species. Beside the theoretical interest in how these external influences may affect pattern-formation mechanisms, there is a practical interest in how a changing climate, as predicted by IPCC (Stocker et al. 2014), may affect species distribution patterns. Integrodifference equations represent the life cycle of organisms with distinct growth and dispersal phases, and thereby may emulate seasonal variation as well as seasonal reproduction (Neubert et al. 1995; Eigentler and Sherratt 2018). Their analysis reveals that pattern formation arises under much milder conditions, e.g. in the absence of an activator-inhibitor structure, if the dispersal patterns are sufficiently different from simple diffusion (Neubert et al. 1995). In integrodifference equations, however, the growth and dispersal processes are completely decoupled and no species interactions occur during the dispersal season.

We use a relatively recent modelling approach to investigate how seasonal variation and discrete reproduction affects diffusion-driven pattern formation. Our model considers a twospecies community in two temporal stages: within a year (or 'growing season' or 'summer') 
and between years (or 'dormant season' or 'winter'). The prey/resource grows and diffuses continuously during the year and declines between years. The predator/consumer moves and consumes the prey throughout the year but does not grow in numbers/density. Reproduction of the predator happens at the beginning of the year where the resource accumulated during the previous year is converted to offspring. Our modelling framework builds on previous work by Huang et al. (2017), Lewis and Li (2012), Vasilyeva et al. (2016) and can be classified as a hybrid continuous/discrete model or as an impulsive reaction-diffusion model. Our model is also a generalization of the non-spatial hybrid model proposed by Pachepsky et al. (2008). We give a detailed model derivation in Section 2. The analysis in Section 3 proceeds in two steps: in a simple case, we can explicitly calculate solutions and show that no pattern formation can occur from a linear functional response. In the general case, we use bifurcation results to derive conditions under which pattern formation can occur. In Section 4, we show by numerical simulations that pattern formation does indeed occur and that the order of magnitude separation of dispersal scales is not necessary in our model, provided that certain other conditions are satisfied.

\section{Model formulation}

Different time scales for reproduction between prey/resource and predator/consumer are ubiquitous in ecological communities. Aphids, for example, produce several generations per year, whereas their insect predators usually have only one. Plants grow continually throughout their growing season, whereas some mammal and insect herbivores reproduce only once per year. Hares and other rodents can have several generations per year, whereas lynx and other predators give birth only once. Accordingly, we split one year into two stages and model resource reproduction as continuous throughout the year or at least the growing season within a year. Consumer reproduction, on the other hand, occurs as an impulse at the beginning of a year. The non-spatial dynamics in our model follow closely the model proposed by Pachepsky et al. (2008), where still more ecological examples for this separation of scales can be found.

The challenge with this modelling approach is that since consumed resource is not immediately converted to 'active' consumers, we need to keep track of consumed resources separately, as was done by Geritz and Kisdi (2004) and Pachepsky et al. (2008) in a non-spatial setting. Hence, our model has three compartments: resource $(F)$, consumer $(C)$, and 'consumed biomass' or

'internal stored energy' $(E)$. Stored energy decreases due to the death and metabolism of the consumer throughout the year. As is common in studies of pattern formation, we model spatial movement by diffusion.

We denote by $n=0,1, \ldots$ the year and by $0 \leq t \leq T$ time within each year. The spatial location is denoted by $x$. Accordingly, we write $F_{n}(x, t)$ for the density of the resource, $C_{n}(x, t)$ 
for the density of the consumer, and $E_{n}(x, t)$ for the total stored energy of the consumer at location $x$ and time $t$ within the $n^{\text {th }}$ year.

The model equations for the within-year dynamics $(0 \leq t \leq T)$ are given by

$$
\begin{aligned}
& \frac{\partial F_{n}(x, t)}{\partial t}=g\left(F_{n}\right)-h\left(F_{n}, C_{n}\right) C_{n}+d \frac{\partial^{2} F_{n}}{\partial x^{2}}, \\
& \frac{\partial C_{n}(x, t)}{\partial t}=-m C_{n}+D \frac{\partial^{2} C_{n}}{\partial x^{2}}, \\
& \frac{\partial E_{n}(x, t)}{\partial t}=\gamma h\left(F_{n}, C_{n}\right) C_{n}-(m+\delta) E_{n}+D \frac{\partial^{2} E_{n}}{\partial x^{2}} .
\end{aligned}
$$

Function $g$ denotes the growth function of the resource throughout a year, whereas $h$ is the functional response per-capita of predator. Parameters $\gamma$ and $m$ are the conversion efficiency and consumer mortality, respectively. Total stored energy declines throughout a year with rate $m+\delta$, the sum of consumer death with rate $m$ and metabolism with rate $\delta$. The diffusion coefficients for resource and consumer are $d$ and $D$, respectively. Since resource is consumed rather than cached, stored energy has the exact same movement behavior as consumers. Hence, the usual derivation of the diffusion equation from a random walk for the consumer also applies to each unit of stored energy. Therefore, the two have the same diffusion coefficient. A similar modelling approach can also be found in the article by Hsu et al. (2010), where a spatial version of the Droop model was proposed in a chemostat environment.

We point out that it is possible in our model that the net flux of consumers is zero $(D \partial C / \partial x=$ $0)$ while the net flux of stored energy is not $(D \partial E / \partial x \neq 0)$, and vice versa, as it should be. For example, if the density of consumers is constant throughout the domain but consumers in some areas carry more stored energy than in other areas, then the random walk of the individual consumers will tend to equalize the distribution of stored energy even though the net movement of consumers is zero. If we had chosen to model energy as per capita stored energy $(B=E / C)$ rather than total energy $(E)$, as was done by Pachepsky et al. (2008) in their non-spatial model, our spatial model would have looked more complicated due to additional flux terms resulting from differentiating the quotient $B=E / C$.

For our analysis, space is a bounded interval $[0, L]$, but for illustrative simulations, we use a two-dimensional square domain. In both cases, we impose no-flux boundary conditions. In the case of the interval, these are

$$
\frac{\partial F_{n}(0, t)}{\partial x}=0, \quad \frac{\partial F_{n}(L, t)}{\partial x}=0
$$

for the resource and similarly for $C$ and $E$.

In our examples, we will use the logistic growth function for $g$. For the functional response, we will use several of the many well-known forms (Beddington 1975; Holling 1959a; Holling 1959b), and explain how the precise choice will determine whether or not pattern formation is possible. 
We formulate the dynamics between years as an impulse or reset as difference equation. The resource will not increase but may decline, depending on climatic and other conditions. The total stored energy of consumers will be converted to offspring at the beginning of the following year. The stored energy is then set to zero. The equations that relate the population densities at the end of one year to the beginning of the next are

$$
\begin{aligned}
& F_{n+1}(x, 0)=\rho F_{n}(x, T), \\
& C_{n+1}(x, 0)=\sigma E_{n}(x, T)+C_{n}(x, T), \\
& E_{n+1}(x, 0)=0,
\end{aligned}
$$

where $\sigma$ represents the reproduction rate of the consumer, and $0 \leq \rho \leq 1$ is the fraction of resource that survives from the end of one growing season to the beginning of the next. We assumed a linear conversion from stored energy to consumer density. Such an assumption is implicitly made in most consumer resource systems when internal energy is not explicitly modeled. More complicated nonlinear relationships can easily be incorporated into our model. The abstract analysis remains the same but explicit calculations become harder or impossible.

\section{Mathematical analysis}

The classical approach to study diffusion-driven pattern formation is to find a spatially homogeneous steady state that is asymptotically stable, linearize the model at that state, and find conditions under which at least one of the spatial modes becomes unstable. We follow the same steps, except that we cannot expect there to be a positive steady state because of the impulse condition in the model. Instead, we will find a spatially homogeneous impulsive periodic orbit, linearize at this orbit and find conditions such that at least one spatial mode becomes unstable.

We proceed in two steps. We begin with the simple linear functional response, for which we can explicitly calculate the spatially homogeneous periodic orbit and the stability conditions. However, we find that pattern formation is impossible in this case. Then we look at a general functional response and derive sufficient conditions for pattern formation, which we illustrate with a Beddington-DeAngelis functional response.

\subsection{Linear functional response}

We use logistic growth of the resource and the simplest linear functional response

$$
g(F)=r\left(1-\frac{F}{K}\right) F, \quad h(F)=a F .
$$


Here $r$ is the intrinsic growth rate of the resource, $K$ is the carrying capacity of the environment, and $a$ represents the attack rate. We non-dimensionalize system (2.1), (2.3) by taking

$$
F_{n}=K \tilde{F}_{n}, \quad C_{n}=\frac{1}{a T} \tilde{C}_{n}, \quad E_{n}=\frac{1}{a T \sigma} \tilde{E}_{n}, \quad t=T \tilde{t}, \quad x=\sqrt{d T} \tilde{x} .
$$

After dropping $\sim$ for notational convenience, the re-scaled equations are

$$
\begin{array}{ll}
\frac{\partial F_{n}(x, t)}{\partial t}=r_{1}\left(1-F_{n}\right) F_{n}-F_{n} C_{n}+\frac{\partial^{2} F_{n}}{\partial x^{2}} & \\
\frac{\partial C_{n}(x, t)}{\partial t}=-m_{1} C_{n}+D_{1} \frac{\partial^{2} C_{n}}{\partial x^{2}}, & 0 \leq t \leq 1 \\
\frac{\partial E_{n}(x, t)}{\partial t}=\beta F_{n} C_{n}-\delta_{1} E_{n}+D_{1} \frac{\partial^{2} E_{n}}{\partial x^{2}} &
\end{array}
$$

and

$$
\begin{aligned}
& F_{n+1}(x, 0)=\rho F_{n}(x, 1), \\
& C_{n+1}(x, 0)=E_{n}(x, 1)+C_{n}(x, 1), \\
& E_{n+1}(x, 0)=0,
\end{aligned}
$$

respectively. Here, $r_{1}=r T, \beta=\gamma a K T \sigma, m_{1}=m T, D_{1}=D / d, \delta_{1}=(m+\delta) T$ in (3.3). The boundary conditions (2.2) remain unchanged, but the length of the domain is re-scaled to $l=$ $L /(\sqrt{d T})$.

To study spatially homogeneous solutions, we drop the diffusion terms and write the densities as $\left(F_{n}(t), C_{n}(t), E_{n}(t)\right)$. The solution of the consumer equation in (3.3) with initial conditions $C_{n}(0)$ is

$$
C_{n}(t)=C_{n}(0) e^{-m_{1} t} \quad \text { for } \quad 0 \leq t \leq 1
$$

so that

$$
C_{n}(1)=C_{n}(0) e^{-m_{1}}
$$

gives the consumer density at the end of the $n^{\text {th }}$ year.

The equation for the resource is a time-dependent logistic equation that can be solved by standard means to obtain

$$
F_{n}(t)=\frac{F_{n}(0) \kappa(t)}{1+r_{1} F_{n}(0) \int_{0}^{t} \kappa(s) d s},
$$

where $F_{n}(0)$ is the initial density and

$$
\kappa(t)=\kappa\left(t, C_{n}(0)\right)=e^{r_{1} t+\left(C_{n}(0) / m_{1}\right)\left(e^{-m_{1} t}-1\right)} .
$$


In particular, we obtain the resource density at the end of the $n^{\text {th }}$ year as

$$
F_{n}(1)=\frac{F_{n}(0) \kappa(1)}{1+r_{1} F_{n}(0) \int_{0}^{1} \kappa(s) d s} .
$$

Finally, we solve the equation for $E_{n}(t)$ in (3.3) and substitute $F_{n}(t), C_{n}(t)$ from (3.7) and (3.5) to obtain

$$
\begin{aligned}
E_{n}(t) & =E_{n}(0) e^{-\delta_{1} t}+\beta e^{-\delta_{1} t} \int_{0}^{t} e^{\delta_{1} s} F_{n}(s) C_{n}(s) d s \\
& =\beta e^{-\delta_{1} t} \int_{0}^{t}\left[\frac{e^{\delta_{1} s} F_{n}(0) \kappa(s) C_{n}(0) e^{-m_{1} s}}{1+r_{1} F_{n}(0) \int_{0}^{s} \kappa(\eta) d \eta}\right] d s
\end{aligned}
$$

where $E_{n}(0)=0$ from (3.4). Again, the total stored energy at the end of the $n^{\text {th }}$ year is

$$
E_{n}(1)=\beta e^{-\delta_{1}} \int_{0}^{1}\left[\frac{F_{n}(0) C_{n}(0) e^{\delta_{1} s} \kappa(s) e^{-m_{1} s}}{1+r_{1} F_{n}(0) \int_{0}^{s} \kappa(\eta) d \eta}\right] d s
$$

We substitute (3.6), (3.9), and (3.11) into the between-year equations (3.4) to obtain the map from the beginning of one year to the next as

$$
\begin{aligned}
F_{n+1}(0) & =\frac{\rho F_{n}(0) \kappa\left(1, C_{n}(0)\right)}{1+r_{1} F_{n}(0) \int_{0}^{1} \kappa\left(s, C_{n}(0)\right) d s} \\
C_{n+1}(0) & =C_{n}(0) e^{-m_{1}}+\beta \int_{0}^{1}\left[\frac{e^{\delta_{1}(s-1)} F_{n}(0) \kappa\left(s, C_{n}(0)\right) C_{n}(0) e^{-m_{1} s}}{1+r_{1} F_{n}(0) \int_{0}^{s} \kappa\left(\eta, C_{n}(0)\right) d \eta}\right] d s .
\end{aligned}
$$

The fixed points of this system correspond to the densities at the beginning of a year in an impulsive periodic orbit.

We obtain an extinction fixed point $P_{b}=(0,0)$, which always exists, and a semi-trivial fixed point

$$
P_{s}=\left(\frac{\rho e^{r_{1}}-1}{e^{r_{1}}-1}, 0\right) \quad \text { if } \quad e^{r_{1}}>\frac{1}{\rho} .
$$

Moreover, a positive fixed point $P=(\bar{F}, \bar{C})$ is determined by the pair of equations

$$
\left\{\begin{array}{l}
\frac{\rho \kappa(1, \bar{C})}{1+r_{1} \bar{F} \int_{0}^{1} \kappa(s, \bar{C}) d s}=1 \\
\beta e^{-\delta_{1}} \int_{0}^{1}\left[\frac{e^{\left(\delta_{1}-m_{1}\right) s} \bar{F} \kappa(s, \bar{C})}{1+r_{1} \bar{F} \int_{0}^{s} \kappa(\eta, \bar{C}) d \eta}\right] d s=1-e^{-m_{1}}
\end{array}\right.
$$

These equations can be simplified somewhat by solving the first equation as

$$
\bar{F}=\frac{\rho \kappa(1, \bar{C})-1}{r_{1} \int_{0}^{1} \kappa(s, \bar{C}) d s}
$$


and substituting to obtain the implicit equation for $\bar{C}$ as

$$
\int_{0}^{1}\left[\frac{e^{\left(\delta_{1}-m_{1}\right) s} \kappa(s, \bar{C})}{\int_{0}^{s} \kappa(\tau, \bar{C}) d \tau+\left(\int_{0}^{1} \kappa(\tau, \bar{C}) d \tau\right) /(\rho \kappa(1, \bar{C})-1)}\right] d s=\frac{r_{1}\left(e^{m_{1}}-1\right) e^{\delta_{1}}}{\beta e^{m_{1}}} .
$$

For the special case where $\delta_{1}=m_{1}$, i.e. $\delta=0$, we find the explicit expression (see also Pachepsky et al. 2008)

$$
\bar{C}=\frac{m_{1}\left(r_{1}+\ln \rho-\left(r_{1} / \beta\right)\left(e^{m_{1}}-1\right)\right)}{1-e^{-m_{1}}}
$$

provided

$$
r_{1}>\frac{\beta \ln \rho}{e^{m_{1}}-1-\beta}>0
$$

To summarize, we found up to three spatially homogeneous, impulsive orbits in our system. The trivial one is actually a classical equilibrium solution

$$
F_{n}(t)=0, \quad C_{n}(t)=0, \quad E_{n}(t)=0 \quad \text { for } \quad 0 \leq t \leq 1, \quad n=0,1,2, \ldots
$$

The semi-trivial fixed point $P_{s}$, if it exists, leads to the impulsive periodic solution

$$
F_{n}(t)=\frac{\left(\rho e^{r_{1}}-1\right) \kappa(t)}{e^{r_{1}}-1+r_{1}\left(\rho e^{r_{1}}-1\right) \int_{0}^{t} \kappa(s) d s}, \quad C_{n}(t)=0, \quad E_{n}(t)=0, \quad \text { for } \quad 0 \leq t \leq 1
$$

with $\kappa$ as in (3.8). Similarly, if the positive fixed point $P$ exists, it corresponds to an impulsive orbit with all three compartments being positive.

We now study the local stability of these impulsive orbits among all spatially homogeneous solutions. Let $\tilde{F}_{n}(t), \tilde{C}_{n}(t), \tilde{E}_{n}(t)$ be a small perturbation of system (3.3) at the impulsive periodic solution $\left(F_{n}(t), C_{n}(t), E_{n}(t)\right)$. Then the linearization of the within-season system (3.3) is

$$
\frac{d \tilde{\omega}_{n}(t)}{d t}=J_{1} \tilde{\omega}_{n}(t)
$$

where

$$
\tilde{\omega}_{n}(t)=\left(\begin{array}{c}
\tilde{F}_{n}(t) \\
\tilde{C}_{n}(t) \\
\tilde{E}_{n}(t)
\end{array}\right), \quad J_{1}=\left(\begin{array}{ccc}
r_{1}-2 r_{1} F_{n}(t)-C_{n}(t) & -F_{n}(t) & 0 \\
0 & -m_{1} & 0 \\
\beta C_{n}(t) & \beta F_{n}(t) & -\delta_{1}
\end{array}\right) .
$$

Similarly, the linearization of the between-year updates leads to

$$
\tilde{\omega}_{n+1}(0)=J_{2} \tilde{\omega}_{n}(1)
$$

where

$$
J_{2}=\left(\begin{array}{lll}
\rho & 0 & 0 \\
0 & 1 & 1 \\
0 & 0 & 0
\end{array}\right)
$$


It turns out that we can again successively solve our system. The equation for $\tilde{C}_{n}$ has the exponential solution $\tilde{C}_{n}(t)=\tilde{C}_{n}(0) e^{-m_{1} t}$. After substituting this solution into the equation for $\tilde{F}_{n}$, we can solve explicitly as

$$
\tilde{F}_{n}(t)=f_{11}(t) \tilde{F}_{n}(0)+f_{12}(t) \tilde{C}_{n}(0)
$$

where

$$
f_{11}(t)=e^{\int_{0}^{t}\left[r_{1}-2 r_{1} F_{n}(s)-C_{n}(s)\right] d s}, \quad f_{12}(t)=-\int_{0}^{t}\left[F_{n}(s) e^{-m_{1} s} e^{\int_{s}^{t}\left[r_{1}-2 r_{1} F_{n}(\eta)-C_{n}(\eta)\right] d \eta}\right] d s .
$$

Plugging (3.24) into the equation for $\tilde{E}_{n}$ and solving gives

$$
\tilde{E}_{n}(t)=g_{11}(t) \tilde{F}_{n}(0)+g_{12}(t) \tilde{C}_{n}(0)+e^{-\delta_{1} t} \tilde{E}_{n}(0)
$$

where

$$
g_{11}(t)=\beta e^{-\delta_{1} t} \int_{0}^{t}\left[e^{\delta_{1} s} f_{11}(s) C_{n}(s)\right] d s, \quad g_{12}(t)=\beta e^{-\delta_{1} t} \int_{0}^{t} e^{\delta_{1} s}\left[f_{12}(s) C_{n}(s)+F_{n}(s) e^{-m_{1} s}\right] d s .
$$

Now we set $t=1$ to obtain $\tilde{C}_{n}(1), \tilde{F}_{n}(1)$, and $\tilde{E}_{n}(1)$. Substituting these values into (3.23) and rearranging terms leads to

$$
\left(\begin{array}{c}
\tilde{F}_{n+1}(0) \\
\tilde{C}_{n+1}(0) \\
\tilde{E}_{n+1}(0)
\end{array}\right)=\left(\begin{array}{ccc}
J_{11}^{0} & J_{12}^{0} & 0 \\
J_{21}^{0} & J_{22}^{0} & J_{23}^{0} \\
0 & 0 & 0
\end{array}\right)\left(\begin{array}{c}
\tilde{F}_{n}(0) \\
\tilde{C}_{n}(0) \\
\tilde{E}_{n}(0)
\end{array}\right)
$$

where

$$
\begin{aligned}
J_{11}^{0} & =\rho e^{\int_{0}^{1}\left[r_{1}-2 r_{1} F_{n}(s)-C_{n}(s)\right] d s}, \quad J_{12}^{0}=-\rho \int_{0}^{1}\left[F_{n}(s) e^{-m_{1} s} e^{\int_{s}^{1}\left[r_{1}-2 r_{1} F_{n}(\xi)-C_{n}(\xi)\right] d \xi}\right] d s \\
J_{21}^{0} & =\beta e^{-\delta_{1}} \int_{0}^{1}\left[C_{n}(s) e^{\delta_{1} s} e^{\int_{0}^{s}\left[r_{1}-2 r_{1} F_{n}(\xi)-C_{n}(\xi)\right] d \xi}\right] d s \\
J_{22}^{0} & =\beta e^{-\delta_{1}} \int_{0}^{1}\left\{F_{n}(s) e^{\left(\delta_{1}-m_{1}\right) s}-C_{n}(s) e^{\delta_{1} s} \int_{0}^{s}\left[F_{n}(\eta) e^{-m_{1} \eta} e^{\int_{\eta}^{s}\left[r_{1}-2 r_{1} F_{n}(\xi)-C_{n}(\xi)\right] d \xi}\right] d \eta\right\} d s \\
& +e^{-m_{1}}, \quad J_{23}^{0}=e^{-\delta_{1}} .
\end{aligned}
$$

Hence, we can give necessary and sufficient conditions for the local asymptotic stability of our space-independent solutions.

Proposition 3.1. The impulsive periodic solution $\left(F_{n}(t), C_{n}(t), E_{n}(t)\right)$ is locally asymptotically stable if all three eigenvalues $\lambda_{i}^{0}$ of the matrix $J^{0}=\left(J_{i j}^{0}\right)$ lie within the unit circle, or equivalently 
if the three Jury conditions

$$
\left\{\begin{array}{l}
1+J_{11}^{0} J_{22}^{0}-J_{12}^{0} J_{21}^{0}-\left(J_{11}^{0}+J_{22}^{0}\right)>0 \\
1+J_{11}^{0} J_{22}^{0}-J_{12}^{0} J_{21}^{0}+\left(J_{11}^{0}+J_{22}^{0}\right)>0 \\
1-\left(J_{11}^{0} J_{22}^{0}-J_{12}^{0} J_{21}^{0}\right)>0
\end{array}\right.
$$

hold.

For the trivial solution (3.18), the eigenvalues can be calculated explicitly as

$$
\lambda_{1}^{0}=\rho e^{r_{1}}, \quad \lambda_{2}^{0}=e^{-m_{1}}, \quad \lambda_{3}^{0}=0 .
$$

Hence, the trivial solution is stable if $\rho e^{r_{1}}<1$ and unstable if $\rho e^{r_{1}}>1$.

For the semi-trivial impulsive periodic solution (3.19), we obtain eigenvalues

$$
\lambda_{1}^{0}=\rho e^{r_{1} \int_{0}^{1}\left[1-2 F_{s}(s)\right] d s}, \quad \lambda_{2}^{0}=e^{-m_{1}}+e^{-\delta_{1}} \beta \int_{0}^{1}\left[F_{n}(s) e^{\left(\delta_{1}-m_{1}\right) s}\right] d s, \quad \lambda_{3}^{0}=0 .
$$

Clearly, $\left|\lambda_{3}^{0}\right|<1$. We show that $\left|\lambda_{1}^{0}\right|<1$ as well. We see that

$$
\begin{aligned}
\left|\lambda_{1}^{0}\right|<1 & \Leftrightarrow e^{r_{1} \int_{0}^{1}\left(1-2 F_{n}(s)\right) d s}<\frac{1}{\rho} \Leftrightarrow \int_{0}^{1}\left(1-2 F_{n}(s)\right) d s<\frac{1}{r_{1}} \ln \left(\frac{1}{\rho}\right) \\
& \Leftrightarrow-\frac{1}{r_{1}}\left[2 \ln \left(1-F_{n}(0)+e^{r_{1}} F_{n}(0)\right)-r_{1}\right]<\frac{1}{r_{1}} \ln \left(\frac{1}{\rho}\right) \\
& \Leftrightarrow \rho e^{r_{1}}<\left[1-F_{n}(0)+e^{r_{1}} F_{n}(0)\right]^{2} \Leftrightarrow 0<\rho e^{r_{1}}-1,
\end{aligned}
$$

which is automatically satisfied if $P_{s}$ exists. The integral in $\lambda_{2}^{0}$ is positive but cannot be calculated explicitly. Depending on parameters, $\lambda_{2}^{0}$ can be larger or smaller than 1 . Hence, we conclude that the impulsive periodic solution (3.19) is stable if $\lambda_{2}^{0}<1$ and is unstable if $\lambda_{2}^{0}>1$.

Remark 3.2. For the special case where $\delta_{1}=m_{1}, \lambda_{2}^{0}$ can be calculated explicitly as

$$
\lambda_{2}^{0}=e^{-m_{1}}+\frac{\beta e^{-m_{1}}}{r_{1}}\left(r_{1}+\ln \rho\right) .
$$

Hence, the impulsive periodic solution (3.19) is locally asymptotically stable if

$$
\beta\left(1+\frac{\ln (\rho)}{r_{1}}\right)<e^{m_{1}}-1
$$

and unstable if this inequality is reversed.

At the bifurcation point, the eigenvalue satisfies $\lambda_{2}^{0}=1$. Given the biological interpretation and the behavior of most predator-prey systems, we expect a transcritical bifurcation to occur. A transcritical bifurcation for the discrete-time map corresponds to the emergence of a positive 
impulsive periodic orbit in the seasonal model. Hence, we conjecture that a positive impulsive periodic solution (3.5), (3.7), and (3.10) with initial conditions given by (3.13) exists if

$$
e^{-m_{1}}+\beta e^{-\delta_{1}} \int_{0}^{1}\left[\frac{\left(\rho e^{r_{1}}-1\right) e^{\left(r_{1}+\delta_{1}-m_{1}\right) s}}{e^{r_{1}}-1+\left(\rho e^{r_{1}}-1\right)\left(e^{r_{1} s}-1\right)}\right] d s>1 .
$$

Finally, we analyze possible pattern formation from a spatially homogeneous, impulsive periodic solution. Hence, we linearize again at such a solution, but this time with regards to spatially non-constant perturbations. As above, we linearize the within-year system (3.3) and the between-year system (3.4) separately. The linearization of (3.3) at the spatially homogeneous solution $\left(F_{n}(t), C_{n}(t), E_{n}(t)\right)$ is

$$
\begin{aligned}
& \frac{\partial \tilde{\omega}_{n}(x, t)}{\partial t}=\mathcal{D} \frac{\partial^{2} \tilde{\omega}_{n}(x, t)}{\partial x^{2}}+J_{1} \tilde{\omega}_{n}(x, t), \\
& \frac{\partial \tilde{\omega}_{n}(0, t)}{\partial x}=0, \quad \frac{\partial \tilde{\omega}_{n}(l, t)}{\partial x}=0
\end{aligned}
$$

where $J_{1}$ is as in $(3.21)$ and

$$
\tilde{\omega}_{n}(x, t)=\left(\begin{array}{c}
\tilde{F}_{n}(x, t) \\
\tilde{C}_{n}(x, t) \\
\tilde{E}_{n}(x, t)
\end{array}\right), \quad \mathcal{D}=\left(\begin{array}{ccc}
1 & 0 & 0 \\
0 & D_{1} & 0 \\
0 & 0 & D_{1}
\end{array}\right) .
$$

The linearization of the between-year updates (3.4) is

$$
\tilde{\omega}_{n+1}(x, 0)=J_{2} \tilde{\omega}_{n}(x, 1)
$$

where $J_{2}$ is as in (3.23).

We first solve the (linear) consumer equation by the method of separating variables (Logan 2014; Murray 2001). Direct calculations show that

$$
\tilde{C}_{n}(x, t)=\sum_{m=0}^{\infty} \bar{C}_{m} e^{\left[-D_{1}\left(\frac{m \pi}{l}\right)^{2}-m_{1}\right] t} \cos \left(\frac{m \pi x}{l}\right)
$$

where

$$
\bar{C}_{m}=\left\{\begin{array}{l}
\frac{1}{l} \int_{0}^{l} \tilde{C}_{n}(x, 0) d x:=\tilde{C}_{n}(0) \quad \text { if } \quad m=0, \\
\frac{2}{l} \int_{0}^{l} \tilde{C}_{n}(x, 0) \cos \left(\frac{m \pi x}{l}\right) d x \quad \text { if } \quad m \neq 0 .
\end{array}\right.
$$

With this result, we now calculate $\tilde{F}_{n}(x, t)$ from (3.36) and (3.37) by the method of separation of variables and obtain

$$
\tilde{F}_{n}(x, t)=\sum_{m=0}^{\infty}\left[\bar{F}_{m} e^{-\int_{0}^{t} a_{m}(s) d s}-\int_{0}^{t} b_{m}(\eta) e^{\int_{t}^{\eta} a_{m}(s) d s} d \eta\right] \cos \left(\frac{m \pi x}{l}\right),
$$


where

$$
\bar{F}_{m}=\left\{\begin{array}{l}
\frac{1}{l} \int_{0}^{l} \tilde{F}_{n}(x, 0) d x:=\tilde{F}_{n}(0) \quad \text { if } \quad m=0 \\
\frac{2}{l} \int_{0}^{l} \tilde{F}_{n}(x, 0) \cos \left(\frac{m \pi x}{l}\right) d x \quad \text { if } \quad m \neq 0
\end{array}\right.
$$

and

$$
a_{m}(t)=\left(\frac{m \pi}{l}\right)^{2}-\left[r_{1}-2 r_{1} F_{n}(t)-C_{n}(t)\right], \quad b_{m}(t)=F_{n}(t) \bar{C}_{m} e^{\left[-D_{1}\left(\frac{m \pi}{l}\right)^{2}-m_{1}\right] t} .
$$

A final application of the method of separation of variables to the equation for $\tilde{E}_{n}(x, t)$ gives

$$
\tilde{E}_{n}(x, t)=\sum_{m=0}^{\infty}\left[\bar{E}_{m} e^{-\delta_{m} t}+\int_{0}^{t}\left[e^{\delta_{m}(\xi-t)} g_{m}(\xi)\right] d \xi\right] \cos \left(\frac{m \pi x}{l}\right)
$$

where

$$
\bar{E}_{m}=\left\{\begin{array}{l}
\frac{1}{l} \int_{0}^{l} \tilde{E}_{n}(x, 0) d x:=\tilde{E}_{n}(0) \quad \text { if } \quad m=0 \\
\frac{2}{l} \int_{0}^{l} \tilde{E}_{n}(x, 0) \cos \left(\frac{m \pi x}{l}\right) d x \quad \text { if } \quad m \neq 0
\end{array}\right.
$$

and

$$
\begin{aligned}
\delta_{m} & =D_{1}\left(\frac{m \pi}{l}\right)^{2}+\delta_{1}, \quad g_{m}(t)=\bar{F}_{m} \beta C_{n}(t) e^{-\int_{0}^{t} a_{m}(s) d s}+\bar{C}_{m} \beta F_{n}(t) e^{\left[-D_{1}\left(\frac{m \pi}{l}\right)^{2}-m_{1}\right] t} \\
& -\beta C_{n}(t) \int_{0}^{t} b_{m}(\eta) e^{\int_{t}^{\eta} a_{m}(s) d s} d \eta .
\end{aligned}
$$

Substituting $t=1$ into (3.41), (3.39) and (3.44), we obtain $\tilde{F}_{n}(x, 1), \tilde{C}_{n}(x, 1)$ and $\tilde{E}_{n}(x, 1)$, respectively. We notice that the expressions for the different modes $k=(m \pi) / l$ separate. Similarly, the between-year updates (3.38) keep separate modes separately. Hence we can write the expansion

$$
\tilde{C}_{n}(x, 1)=\sum_{k=0}^{\infty} \mathcal{C}_{n}(k) \cos (k x)
$$

with

$$
\mathcal{C}_{n}(k)=\bar{C}_{m} e^{\left[-D_{1}\left(\frac{m \pi}{l}\right)^{2}-m_{1}\right]}
$$

from (3.39) for the consumer, and similarly for the resource and the stored energy. Then the between-year updates (3.38) give us the discrete system for each mode as

$$
\left(\begin{array}{c}
\mathcal{F}_{n+1}(k) \\
\mathcal{C}_{n+1}(k) \\
\mathcal{E}_{n+1}(k)
\end{array}\right)=\left(\begin{array}{ccc}
J_{11}(k) & J_{12}(k) & 0 \\
J_{21}(k) & J_{22}(k) & J_{23}(k) \\
0 & 0 & 0
\end{array}\right)\left(\begin{array}{c}
\mathcal{F}_{n}(k) \\
\mathcal{C}_{n}(k) \\
\mathcal{E}_{n}(k)
\end{array}\right)
$$


where

$$
\begin{aligned}
J_{11}(k) & =J_{11}^{0} e^{-k^{2}}, \quad J_{12}(k)=-\rho \int_{0}^{1}\left[e^{k^{2}\left(\eta-D_{1} \eta-1\right)} F_{n}(\eta) e^{-m_{1} \eta} e^{\left.-\int_{1}^{\eta}\left[r_{1}-2 r_{1} F_{n}(s)-C_{n}(s)\right] d s\right] d \eta,}\right. \\
J_{21}(k) & =\beta \int_{0}^{1}\left[C_{n}(\xi) e^{k^{2}\left(D_{1} \xi-D_{1}-\xi\right)} e^{\delta_{1}(\xi-1)} e^{\left.\int_{0}^{\xi}\left[r_{1}-2 r_{1} F_{n}(s)-C_{n}(s)\right] d s\right] d \xi}\right. \\
J_{22}(k) & =e^{-m_{1}-D_{1} k^{2}}+\beta e^{-\left(D_{1} k^{2}+\delta_{1}\right)} \int_{0}^{1}\left\{F_{n}(\xi) e^{\left(\delta_{1}-m_{1}\right) \xi}-C_{n}(\xi) e^{k^{2} \xi\left(D_{1}-1\right)} e^{\delta_{1} \xi}\right. \\
& \left.\int_{0}^{\xi}\left[F_{n}(\eta) e^{\left(k^{2}-D_{1} k^{2}-m_{1}\right) \eta} e^{-\int_{\xi}^{\eta}\left[r_{1}-2 r_{1} F_{n}(s)-C_{n}(s)\right] d s}\right] d \eta\right\} d \xi, \quad J_{23}(k)=e^{-\left(D_{1} k^{2}+\delta_{1}\right)} .
\end{aligned}
$$

Note that for $k=0$, the entries $J_{i j}(0)=J_{i j}^{0}$ reduce to the entries in (3.29).

Applying the Jury conditions for stability to the above matrix, we obtain the necessary condition for pattern formation of impulsive periodic solutions.

Theorem 3.3. Assume that a spatially homogeneous, periodic orbit of (3.3), (3.4) is stable to homogeneous perturbations, i.e. (3.30) - (3.32) hold. A necessary condition for instability of this orbit and the formation of stable spatial patterns is that the conditions

$$
\left\{\begin{array}{l}
1+J_{11} J_{22}-J_{12} J_{21}-\left(J_{11}+J_{22}\right)<0 \\
1+J_{11} J_{22}-J_{12} J_{21}+\left(J_{11}+J_{22}\right)>0 \\
1>J_{11} J_{22}-J_{12} J_{21}
\end{array}\right.
$$

are satisfied for some mode $k>0$.

We can reason abstractly that pattern formation is impossible from the trivial and semi-trivial orbits that we calculated previously. Since some components of these orbits are zero, pattern formation would imply that these densities take on negative values. Negative densities, however, are impossible since the system preserves positivity. Nonetheless, it is instructive to verify the conditions of Theorem 3.3 explicitly.

Corollary 3.4. Pattern formation cannot occur for impulsive periodic solutions if initial conditions are given by $P_{b}$ or $P_{s}$.

Proof. For the trivial impulsive periodic orbit, we obtained eigenvalues $\lambda_{i}^{0}$ for $i=1,2,3$ in (3.33). We assume that $\left|\lambda_{i}^{0}\right|<1$. Substituting the trivial solution into (3.50) gives the eigenvalues of the community matrix (3.49) as

$$
\lambda_{1}=\rho e^{r_{1}} e^{-k^{2}}=e^{-k^{2}} \lambda_{1}^{0}, \quad \lambda_{2}=e^{-m_{1}} e^{-D_{1} k^{2}}=e^{-D_{1} k^{2}} \lambda_{2}^{0}, \quad \lambda_{3}=0=\lambda_{3}^{0} .
$$

We see that each eigenvalue for mode $k$ can be written as the eigenvalue for mode 0 multiplied by a negative exponential factor of $k^{2}$. In particular, $\left|\lambda_{i}\right|<\left|\lambda_{i}^{0}\right|<1$. Hence, pattern formation cannot occur at the trivial solution. 
The same phenomenon occurs at the semi-trivial impulsive periodic orbit as shown in the following results of the explicit calculations. We obtain

$$
\begin{aligned}
& \left|\lambda_{1}\right|=e^{-k^{2}} \rho e^{\int_{0}^{1}\left[r_{1}-2 r_{1} F_{n}(s)\right] d s}<\lambda_{1}^{0}=\left|\lambda_{1}^{0}\right|<1, \\
& \left|\lambda_{2}\right|=\beta e^{-\left(D_{1} k^{2}+\delta_{1}\right)} \int_{0}^{1}\left[e^{\left(\delta_{1}-m_{1}\right) s} F_{n}(s)\right] d s+e^{-D_{1} k^{2}-m_{1}}<\lambda_{2}^{0}=\left|\lambda_{2}^{0}\right|<1, \\
& \left|\lambda_{3}\right|=0=\left|\lambda_{3}^{0}\right|<1
\end{aligned}
$$

which shows that pattern formation is impossible from the semi-trivial impulsive periodic orbit.

This simplification of separating the Fourier mode $k$ from the eigenvalues $\lambda_{i}^{0}$ or from the entries $J_{i j}^{0}$ of the Jacobian matrix does not arise at a positive impulsive periodic orbit. For that reason, it is impossible to analytically determine explicit conditions for pattern formation at the positive solution from Theorem 3.3. We used extensive numerical simulations to search the parameter space for the model with linear functional response for the possibility of pattern formation. More specifically, we assigned to each parameter in (3.3), (3.4) a wide range of values and picked parameter sets randomly from these ranges. If a set satisfied the condition in (3.35) for the existence of a positive fixed point $P=(\bar{F}, \bar{C})$ as in (3.14), (3.15), we calculated the corresponding orbit $F_{n}(t), C_{n}(t)$ and $E_{n}(t)$. We used this solution to check the pattern formation conditions in Proposition 3.1 and Theorem 3.3. We did this for $10^{7}$ randomly chosen parameter sets. We found that the positive impulsive orbit was stable to spatial non-uniform perturbations whenever it was stable to homogeneous perturbations. We conjecture that the reason for the non-existence of stable spatial patterns is the lack of activator for system (3.3) and (3.4), which is shown in the next proposition.

Proposition 3.5. Suppose that $\left(F_{n}(t), C_{n}(t), E_{n}(t)\right)$ is a homogeneous, positive, impulsive periodic orbit of $(3.3)-(3.4)$. Then $J_{11}^{0}<1$.

Proof.

$$
\begin{aligned}
J_{11}^{0} & =\rho e^{\int_{0}^{1}\left[r_{1}-2 r_{1} F_{n}(s)-C_{n}(s)\right] d s}=\rho e^{r_{1}} e^{-C_{n}(0) \int_{0}^{1} e^{-m_{1} s} d s} e^{-2 \int_{0}^{1}\left[\frac{r_{1} F_{n}(0) \kappa(s)}{1+r_{1} F_{n}(0) \int_{0}^{s} \kappa(\eta) d \eta}\right] d s} \\
& =\rho e^{r_{1}+\left(C_{n}(0) / m_{1}\right)\left(e^{\left.-m_{1}-1\right)}\right.} \frac{1}{\left[1+r_{1} F_{n}(0) \int_{0}^{1} \kappa(\eta) d \eta\right]^{2}}=\frac{1}{\rho \kappa(1)}
\end{aligned}
$$

From the first equation of (3.13), we have

$$
\rho \kappa(1, \bar{C})=1+r_{1} \bar{F} \int_{0}^{1} \kappa(s, \bar{C}) d s>1
$$

because $\bar{F}$ is positive. By combining (3.54) and (3.55), we obtain that $J_{11}^{0}<1$. 


\subsection{Nonlinear functional response}

From the preceding analysis, it is clear that we need to consider nonlinear functional responses if we want to observe pattern formation. Unfortunately, no explicit expressions are available for the positive impulsive periodic orbit in that case. However, we can follow the same procedure as in the previous section to (i) find conditions for the existence of a positive, impulsive orbit; (ii) find the conditions for its stability with respect to homogeneous perturbations, and finally (iii) find conditions for instability with respect to heterogeneous perturbations. We continue to use the logistic growth function from (3.1) for the resource but keep the functional response as general as possible. For illustrations, we use the biologically meaningful Beddington-DeAngelis functional response (Beddington 1975; DeAngelis et al. 1975; Geritz and Gyllenberg 2012)

$$
h(F, C)=\frac{a F}{1+b_{1} F+b_{2} C},
$$

where $a$ represents the attack rate, $b_{1}$ measures the resource-handling time by consumers, and $b_{2}$ represents the intensity of intra-species competition between consumers.

To reduce the number of parameters for explicit calculations, we non-dimensionalize the system $(2.1),(2.2)$ and (2.3) by letting

$$
F_{n}=K \tilde{F}_{n}, \quad C_{n}=b_{1} \tilde{C}_{n}, \quad E_{n}=\frac{b_{1}}{\sigma} \tilde{E}_{n}, \quad t=T \tilde{t}, \quad x=\sqrt{d T} \tilde{x} .
$$

After dropping $^{\sim}$ for notational convenience, we obtain the re-scaled within-year model as

$$
\begin{aligned}
& \frac{\partial F_{n}(x, t)}{\partial t}=r_{1}\left(1-F_{n}\right) F_{n}-\frac{F_{n} C_{n}}{\omega+\theta_{1} F_{n}+\theta_{2} C_{n}}+\frac{\partial^{2} F_{n}}{\partial x^{2}}, \\
& \frac{\partial C_{n}(x, t)}{\partial t}=-m_{1} C_{n}+D_{1} \frac{\partial^{2} C_{n}}{\partial x^{2}}, \quad 0 \leq t \leq 1 \\
& \frac{\partial E_{n}(x, t)}{\partial t}=\frac{\beta F_{n} C_{n}}{\omega+\theta_{1} F_{n}+\theta_{2} C_{n}}-\delta_{1} E_{n}+D_{1} \frac{\partial^{2} E_{n}}{\partial x^{2}},
\end{aligned}
$$

where $r_{1}=r T, \beta=(\gamma K \sigma) / b_{1}, m_{1}=m T, D_{1}=D / d, \delta_{1}=(m+\delta) T, \omega=1 /\left(a b_{1} T\right), \theta_{1}=$ $K /(a T), \theta_{2}=b_{2} /(a T)$. No-flux boundary conditions and the between-year updates remain the same as (2.2) and (3.4), respectively.

In general, the zero solution (3.18) is a solution of system (2.1), (2.2) and (2.3). The stability conditions for this solution depend only on the growth function of the resource, but not on the functional response. Hence, the trivial solution is unstable and a semi-trivial impulsive periodic orbit exists in the general system if $\rho e^{r_{1}}>1$ as above. For the semi-trivial impulsive periodic orbit, the component $F_{n}(t)$ is exactly the same in the general case as calculated in (3.7), i.e. $C_{n}(t)=E_{n}(t)=0$ and

$$
F_{n}(t)=\frac{F_{n}(0) e^{r t}}{1+F_{n}(0)\left(e^{r t}-1\right)}, \quad \text { with } \quad F_{n}(0)=\frac{\rho e^{r_{1}}-1}{e^{r_{1}}-1} .
$$


We expect a positive impulsive orbit to emerge when this semi-tivial orbit becomes unstable. The linearization for a perturbation $\tilde{w}_{n}(t)=\left(\tilde{F}_{n}(t), \tilde{C}_{n}(t), \tilde{E}_{n}(t)\right)$ at this orbit in the non-spatial system is given by

$$
\frac{d}{d t} \tilde{w}_{n}=J_{1} \tilde{w}_{n}, \quad \tilde{w}_{n+1}(0)=J_{2} \tilde{w}_{n}(1)
$$

with $J_{2}$ is the same as $(3.23)$ and

$$
J_{1}=\left(\begin{array}{ccc}
\bar{J}_{11}(t) & \bar{J}_{12}(t) & 0 \\
0 & -m_{1} & 0 \\
\bar{J}_{31}(t) & \bar{J}_{32}(t) & -\delta_{1}
\end{array}\right) .
$$

Calculations similar to the ones in the previous section show that the system decouples and has one eigenvalue equal to zero (corresponding to the $\tilde{E}$ component) and one positive eigenvalue strictly less than unity (corresponding to the $\tilde{F}$ component). The only eigenvalue that may exceed unity corresponds to the consumer component. By the structure of the equations, only the $(2,2)$-entries of each of the matrices determine the persistence conditions for the consumer. In particular, we have

$$
\tilde{C}_{n+1}(0)=\left[e^{-m_{1}}+\beta e^{-\delta_{1}} \int_{0}^{1}\left[e^{\delta_{1} s} h\left(F_{n}(s), 0\right) e^{-m_{1} s}\right] d s\right] \tilde{C}_{n}(0),
$$

so that the consumer can invade the semi-trivial impulsive orbit when

$$
1<e^{-m_{1}}+\beta e^{-\delta_{1}} \int_{0}^{1}\left[e^{\delta_{1} s} h\left(F_{n}(s), 0\right) e^{-m_{1} s}\right] d s .
$$

We expect a positive periodic solution to exist if $\rho e^{r_{1}}>1$ and (3.62) hold. Then we linearize the full spatial system at this solution and require that the linearization be stable with respect to homogeneous perturbations but unstable with respect to non-homogeneous ones, so that patterns may form. To obtain such conditions for a general functional response $h(F, C)$, we linearize system $(2.1)$ at an impulsive periodic solution $\left(F_{n}(t), C_{n}(t), E_{n}(t)\right)$, and obtain

$$
\frac{\partial \tilde{\omega}_{n}(x, t)}{\partial t}=\mathcal{D} \frac{\partial^{2} \tilde{\omega}_{n}(x, t)}{\partial x^{2}}+J_{1} \tilde{\omega}_{n}(x, t)
$$

where $J_{1}$ has the same format as (3.60) and $\mathcal{D}$ is the same as (3.37).

In the above linearization, the entries of $J_{1}$ are

$$
\begin{aligned}
& \bar{J}_{11}(t)=r_{1}-2 r_{1} F_{n}(t)-\frac{\partial h\left(F_{n}, C_{n}\right)}{\partial F_{n}} C_{n}, \quad \bar{J}_{12}(t)=-\left(\frac{\partial h\left(F_{n}, C_{n}\right)}{\partial C_{n}} C_{n}+h\left(F_{n}, C_{n}\right)\right), \\
& \bar{J}_{31}(t)=\beta \frac{\partial h\left(F_{n}, C_{n}\right)}{\partial F_{n}} C_{n}, \quad \bar{J}_{32}(t)=\beta\left[\frac{\partial h\left(F_{n}, C_{n}\right)}{\partial C_{n}} C_{n}+h\left(F_{n}, C_{n}\right)\right] .
\end{aligned}
$$


Again, linearization of $(2.3)$ at $\left(F_{n}(0), C_{n}(0), E_{n}(0)\right)$ gives

$$
\tilde{\omega}_{n+1}(x, 0)=J_{2} \tilde{\omega}_{n}(x, 1)
$$

where $J_{2}$ is the same as $(3.23)$.

Separating variables introduces the exact same expansions of the perturbation functions and introduces the modes $k$ as above. Following the same steps as in the previous section, the equations for the different modes separate, and we can write the linear system for the modes in the exact same form as (3.49). The coefficients are now given by

$$
\begin{aligned}
J_{11} & =\rho e^{-k^{2}} e^{\int_{0}^{1} \bar{J}_{11}\left(F_{n}(s), C_{n}(s)\right) d s}, \\
J_{12} & =\rho \int_{0}^{1}\left[e^{k^{2}\left(\eta-1-D_{1} \eta\right)} \bar{J}_{12}\left(F_{n}(\eta), C_{n}(\eta)\right) e^{-m_{1} \eta} e^{\int_{\eta}^{1} \bar{J}_{11}\left(F_{n}(s), C_{n}(s)\right) d s}\right] d \eta, \\
J_{21} & =\int_{0}^{1}\left[e^{k^{2}\left(D_{1} \eta-D_{1}-\eta\right)} e^{\delta_{1}(\eta-1)} \bar{J}_{31}\left(C_{n}(\eta), F_{n}(\eta)\right) e^{\int_{0}^{\eta} \bar{J}_{11}\left(F_{n}(s), C_{n}(s)\right) d s}\right] d \eta, \\
J_{22} & =e^{\left(-D_{1} k^{2}-m_{1}\right)}+e^{-\left(D_{1} k^{2}+\delta_{1}\right)} \int_{0}^{1}\left\{e^{\left(D_{1} k^{2}+\delta_{1}\right) \xi} \bar{J}_{31}\left(C_{n}(\xi), F_{n}(\xi)\right)\right. \\
& \int_{0}^{\xi}\left[\bar{J}_{12}\left(F_{n}(\eta), C_{n}(\eta)\right) e^{k^{2}\left(\eta-\xi-D_{1} \eta\right)} e^{-m_{1} \eta} e^{-\int_{\xi}^{\eta} \bar{J}_{11}\left(F_{n}(s), C_{n}(s)\right) d s}\right] d \eta \\
& \left.+\bar{J}_{32}\left(F_{n}(\xi), C_{n}(\xi)\right) e^{\left(\delta_{1}-m_{1}\right) \xi}\right\} d \xi, \\
J_{23} & =e^{-D_{1} k^{2}-\delta_{1}} .
\end{aligned}
$$

Hence, for a given functional response, one determines the conditions for the existence of a positive, homogeneous orbit, computes that orbit numerically, and substitutes the result into the above expressions to find the conditions for stability or instability of mode $k$.

We return to our example of the Beddington-DeAngelis functional response. The explicit forms of the entries of matrix $J_{1}$ in $(3.60)$ are

$$
\begin{aligned}
\bar{J}_{11}(t)= & -\frac{1}{\left(C_{n}(t) \theta_{2}+F_{n}(t) \theta_{1}+\omega\right)^{2}}\left[2 C_{n}(t)^{2} F_{n}(t) r_{1} \theta_{2}^{2}+4 C_{n}(t) F_{n}(t)^{2} r_{1} \theta_{1} \theta_{2}+2 F_{n}(t)^{3} r_{1} \theta_{1}^{2}\right. \\
& -C_{n}(t)^{2} r_{1} \theta_{2}^{2}+4 C_{n}(t) F_{n}(t) \omega r_{1} \theta_{2}-2 C_{n}(t) F_{n}(t) r_{1} \theta_{1} \theta_{2}+4 F_{n}(t)^{2} \omega r_{1} \theta_{1}-F_{n}(t)^{2} r_{1} \theta_{1}^{2} \\
& \left.-2 C_{n}(t) \omega r_{1} \theta_{2}+2 F_{n}(t) \omega^{2} r_{1}-2 F_{n}(t) \omega r_{1} \theta_{1}+C_{n}(t)^{2} \theta_{2}-\omega^{2} r_{1}+C_{n}(t) \omega\right] \\
\bar{J}_{12}(t)= & -\frac{F_{n}(t)\left(F_{n}(t) \theta_{1}+\omega\right)}{\left(C_{n}(t) \theta_{2}+F_{n}(t) \theta_{1}+\omega\right)^{2}}, \quad \bar{J}_{31}(t)=\frac{\beta C_{n}(t)\left(C_{n}(t) \theta_{2}+\omega\right)}{\left(C_{n}(t) \theta_{2}+F_{n}(t) \theta_{1}+\omega\right)^{2}} \\
\bar{J}_{32}(t)= & \frac{\beta F_{n}(t)\left(F_{n}(t) \theta_{1}+\omega\right)}{\left(C_{n}(t) \theta_{2}+F_{n}(t) \theta_{1}+\omega\right)^{2}} .
\end{aligned}
$$

By substituting (3.65) into (3.64), we then obtained the corresponding entries, which also determine the stability of the impulsive periodic solutions. 
The statements of Proposition 3.1 and Theorem 3.3 continue to hold with these expressions for the matrix entries in our Beddington-DeAngelis response. Before we proceed to present examples of pattern formation, we first give the proposition below, which shows that for a general resource growth function and a general predation function, spatial pattern formation cannot occur if the diffusion rate of the resource is equal to the diffusion rate of the consumer.

Proposition 3.6. For a general growth term $g(F)$ and general predation term $h(F, C)$, pattern formation cannot occur if the diffusion coefficients of the consumer and the resource are equal, i.e. $D_{1}=1$.

Proof. By substituting $D_{1}=1$ into (3.64), the integrals simplify significantly and we obtain

$$
J_{11}=e^{-k^{2}} J_{11}^{0}, \quad J_{12}=e^{-k^{2}} J_{12}^{0}, \quad J_{21}=e^{-k^{2}} J_{21}^{0}, \quad J_{22}=e^{-k^{2}} J_{22}^{0},
$$

where $J_{i j}^{0}$ for $i, j=1,2$ represent entries of the corresponding community matrix when spatial structure is absent. By Theorem 3.3, we need to check conditions (3.51), (3.52) and (3.53).

Adding (3.30) and (3.32) yields

$$
2>J_{11}^{0}+J_{22}^{0}
$$

By plugging (3.66) into (3.51), we obtain the equivalent form

$$
0>1+e^{-2 k^{2}}\left(J_{11}^{0} J_{22}^{0}-J_{12}^{0} J_{21}^{0}\right)-e^{-k^{2}}\left(J_{11}^{0}+J_{22}^{0}\right) .
$$

Multiplying (3.30) by $e^{-2 k^{2}}$ leads to

$$
e^{-2 k^{2}}+e^{-2 k^{2}}\left(J_{11}^{0} J_{22}^{0}-J_{12}^{0} J_{21}^{0}\right)-e^{-2 k^{2}}\left(J_{11}^{0}+J_{22}^{0}\right)>0 .
$$

Now, adding (3.68) and (3.69) and simplifying the expressions gives

$$
\begin{aligned}
& e^{-2 k^{2}}-e^{-2 k^{2}} J_{11}^{0}-e^{-2 k^{2}} J_{22}^{0}>1-e^{-k^{2}} J_{11}^{0}-e^{-k^{2}} J_{22}^{0} \\
& \Leftrightarrow J_{11}^{0}+J_{22}^{0}>\frac{1-e^{-2 k^{2}}}{e^{-k^{2}}\left(1-e^{-k^{2}}\right)}=1+e^{k^{2}} .
\end{aligned}
$$

From (3.67) and (3.70), we have

$$
2>J_{11}^{0}+J_{22}^{0}>1+e^{k^{2}} \Leftrightarrow 1>e^{k^{2}}
$$

which leads to a contradiction because $k>0$ here. Therefore, pattern formation cannot occur if $D_{1}=1$. 


\section{Numerical simulation}

In this section, we illustrate the theory of pattern formation in impulsive systems using the Beddington-DeAngelis functional response as in (3.57) with between-year updates from (3.4). Figure 1 shows the densities $F_{n}(t), C_{n}(t), E_{n}(t)$ as they converge to a spatially homogeneous positive impulsive orbit. Since the numerical solutions converge to the impulsive orbit, it is clear that the orbit is stable in the non-spatial setting. We observe that the consumer density decreases during each season, whereas the total stored energy and the resource increase. At the beginning of the next season, the consumer density increases due to the births in the population, the energy stored is reset to zero, and the resource decays due to overwinter mortality.

Next, we visualise the pattern-formation conditions (3.51), (3.52), (3.53) as a function of the spatial mode to determine whether and for which modes we might see instability. Based on the analytical results, we choose the diffusion rate for the consumer (inhibitor) larger than for the prey (activator), namely $D / d=D_{1}=10>1$. The upper plot demonstrates that the second and third Jury conditions are satisfied for the entire range. The lower plot, however, indicates that there is an intermediate range of $k$ for which the instability condition (3.51) holds, so that the impulsive orbit is unstable with respect to these modes.

Since we simulate the dynamics on a bounded domain, the spatial modes that satisfy the boundary conditions form a discrete set. By choosing the domain large enough, we can typically ensure that one of the admissible modes lies in the interval where (3.51) holds. Consequently, spatially heterogeneous solutions eventually appear if spatial non-uniform perturbations are applied to the spatially homogeneous, impulsive periodic solution.

When the domain is the one-dimensional interval $0 \leq x \leq L$, the spatial modes with noflux boundary conditions $(2.2)$ are given by $k=(m \pi) / L$, for $m=0,1,2, \ldots$ By choosing $L=20, m=3$, we find $k \approx 0.471$, which is located in the interval where (3.51) is satisfied, see Figure 2. Therefore, conditions (3.51), (3.52) and (3.53) are sufficient and necessary conditions for pattern formation for the set of parameters in Figure 2 and $L=20$. Figure 3 confirms that a spatially heterogeneous solution appears if the diffusion coefficient $D_{1}$ is large enough (panel (a)), but the homogeneous solution is stable if $D_{1}$ is small (panel (b)). Moreover, we see that the pattern that emerges finally corresponds to the unstable mode with $m=3$. We only present the temporal evolution of the consumer density; the plots of resource and stored energy are similar.

We also explored and found pattern formation in the two-dimensional square domain $[0 \leq$ $x \leq L] \times[0 \leq y \leq L]$ with $L=40$. The four temporal snapshots in Figure 4 show how the random initial perturbation near the homogeneous steady state becomes coarser and more regular over time and eventually leads to an arrangement of periodically spaced peaks that is constant for the year-to-year map. The consumers are concentrated in the spots; the density between spots 
is lower.

To produce Figure 4, we employed a finite difference scheme to system (3.57) with boundary condition (2.2) (Bradie 2006). We discretized the domain into a spatial lattice with $\Delta x=\Delta y=$ 0.8 and used a central-space 4-point stencil to represent the diffusion operator. Time steps are chosen to be $\Delta t=0.01$, so that the numerical scheme is stable.

Finally, we explore the requirement for the ratio of the diffusion coefficients $D / d=D_{1}$ for pattern formation. In continuous-time models, one requires $D \gg d$ for a Turing bifurcation (Murray 2001). In our impulsive system, Proposition 3.6 still requires $D>d$ for Turing instability, but as Figure 5(a) shows, the requirement is less severe than in the continuous-time case. Specifically, we use the parameter set in Figure 1 but choose $\rho$ as a free parameter and determine numerically the threshold value of $D_{1}=D / d$ required for pattern formation. We see that the threshold value increases with $\rho$. As $\rho$ becomes small, the threshold value decreases to roughly $D_{1} \approx 5$. We note that we cannot take the limit as $\rho \rightarrow 0$ since the consumer cannot persist when $\rho$ is too small.

To explore what effect climate change may have on the propensity for diffusion-driven instability in our impulsive model, we changed the length $T$ of the growing season ('summer'). This parameter does not appear explicitly in the scaled model, but determines the values of the nondimensional parameters indirectly. However, parameter $\rho$ and $D_{1}$ are independent of $T$. Hence, we created a plot like in Figure 5(a) by choosing a larger T. As shown in Figure 5(b), when $T>1$, i.e., the summer is longer than in Figure 5(a), then pattern formation requires larger values of $D_{1}$ for the same value of $\rho$. We also obtained a plot by choosing $0.7 T$, i.e., the summer is even shorter. The plot is similar to figures 5(a) and 5(b) and is thus omitted here. However, by comparing the three figures $(T<1, T=1, T>1$,$) , we see that the range of \rho$ for which the positive impulsive periodic solution exists depends on $T$. If $T$ is small, then the overwinter survival rate must be higher for the resource to persist and support a consumer. Hence, the axes for $\rho$ differ between the three plots, but they overlap.

\section{Conclusion and Discussion}

Since the original discovery of pattern formation through diffusion-driven instabilities by Turing (1952), these ideas have found many applications in biological systems from developmental biology to animal coat patterns (Murray 2001). Some applications to ecological questions exist, such as the patchiness of plankton (Levin and Segel 1976). In the context of population dynamics, Turing's conditions for pattern-formation require that two species interact as predator and prey. However, the most prominent predator-prey model, the Rosenzweig-MacArthur model, does not allow for diffusion driven instability since the prey does not act as an activator (Murray 2001). 
In addition, the order-of-magnitude difference in diffusion coefficients required for instability is not necessarily present in many predator-prey systems.

Within the last few decades, however, several developments brought the ideas of diffusiondriven instabilities back to ecological theory. Empirically, more examples of pattern formation have been documented and experimentally tested by Rietkerk and Van de Koppel (2008), Sheffer et al. (2013). Theoretically, alternative functional responses have been derived, and it was shown by Fasani and Rinaldi (2011), Morozov et al. (2006), that many of these do allow for pattern formation. All these studies concentrated on population growth and interaction dynamics, aiming to find conditions to obtain an activator-inhibitor structure. In a complementary approach, Neubert et al. (1995) showed that if dispersal behavior differs from simple diffusion, pattern formation does not even require an activator-inhibitor structure. Our work adds another perspective to the theory by considering temporal variation in the form of seasonality.

Our motivation is twofold. Many ecosystems show clear seasonal variation in a number of factors that in turn affect population growth, interaction and dispersal. Specifically, many consumer species do not reproduce continuously but only once per year. As a consequence, the location of consumption and reproduction are decoupled. This decoupling suggests time as a mechanism by which consumer dispersal can become 'long range' compared to the resource without actually being an order of magnitude larger than resource dispersal. In other words, a random perturbation of the homogeneous steady state may lead to a local accumulation of the resource, which acts as an activator and supports the consumer. But because consumer reproduction occurs only at the beginning of the next season when the consumer will have moved away from the local resource accumulation, the consumer cannot effectively inhibit the resource and a pattern can form.

Proposition 3.6 shows that pattern formation is still not possible if resource and consumer disperse at the same rate. The evaluation of the instability conditions in Figure 5 demonstrates, however, that pattern formation is possible with the ratio of the diffusion coefficients being as low as 5. The minimum required ratio of the diffusion coefficients is an increasing function of $\rho$, the overwinter survival probability of the resource. Hence, we see that the discontinuity in resource density, induced by seasonality, is another crucial factor in pattern formation. The more the resource decreases during the winter, the more likely diffusion-driven instabilities arise. In temperate systems, this seasonal variation is inherent, so that this mechanism of pattern formation may be more common than previously assumed. In developmental biology, such seasonality is typically absent, however, it would be interesting to design experiments with temporal variation and test our results empirically.

Our research is related to earlier work on pattern formation in temporally varying environments. Since the pattern formation condition in reaction-diffusion equations depends so strongly 
on the ratio of the diffusion coefficients, one can ask what happens when one of the diffusion coefficients (and hence their ratio) varies in time. Sherratt (1995b), inspired by earlier numerical work, used a periodic, piecewise constant diffusion coefficient and explicitly calculated the instability conditions when the period of the diffusion coefficient is much longer than the time scale of kinetics. He found that when the diffusion coefficient has a constant mean value, the temporal variation in the diffusion coefficient may either promote or inhibit Turing bifurcation. Subsequent work considered also time-dependent kinetics (Sherratt 1995a) and other aspects, e.g. Benson et al. (1998). A crucial difference between these works and ours is that we model reproduction of the consumer as a discrete event so that the densities in our model become discontinuous in time.

We found that no pattern formation is possible with the linear functional response, and we could prove that with that response, there is no activator in the system. Hence, we conjecture that the requirement of an activator is still valid for our seasonal system. We used the same computational approach as with the linear functional response to check whether our system with a Holling type II functional response would produce patterns. We searched parameter space randomly for $10^{7}$ points and could not find any evidence for diffusion-driven instabilities. We found that pattern formation is possible with the Beddington-DeAngelis functional response. We speculate that pattern formation can occur in our system when one of the functional responses that allowed pattern formation in a continuous-time model is used by Fasani and Rinaldi (2011), and we expect that also there, the condition on the diffusion coefficients would be relaxed. Our work complements that by Cobbold et al. (2015), who found that spatial variation can - if it induces an appropriate individual-level movement behavior - lead to pattern formation under less stringent conditions on the diffusion coefficients.

On a more technical side, we note the instability condition for the classical reaction-diffusion model can be formulated in terms of the spatial mode $k$ and the entries of the Jacobian matrix of the non-spatial model (Murray 2001). The same simplification arises in the case of integrodifference equations (Neubert et al. 1995). In our case, it is still true that the stability conditions for the different modes decouple. However, each mode interacts with the density along the entire spatially homogeneous impulsive orbit. For that reason, the instability conditions for our model cannot be formulated in terms of the spatial mode and the stability conditions for the non-spatial models. This complication makes the application of our theory more difficult as it requires numerical evaluation of the conditions.

Our second motivation was to study how global change may affect the propensity of consumerresource interactions to form spatial patterns. A general prediction of many climate scenarios is that the 'summer' season in temperate climates will become longer (Stocker et al. 2014). The results in Figure 5 show that as $T$ increases, the conditions for instability become more 
stringent when the overwinter survival rate remains fixed. On the other hand, longer summers mean shorter winters, and - according to the climate scenarios - also milder winters. Hence, we would expect that as $T$ increases, $\rho$ also increases so that the ratio of diffusion coefficients must be much higher for pattern formation. Hence, we speculate that diffusion-driven pattern formation is less likely under future climates. Non-homogeneous environmental conditions are typically associated with ecological opportunities as species can find and fill different niches. A loss of spatially non-homogeneous patterns then could contribute to a loss of biodiversity.

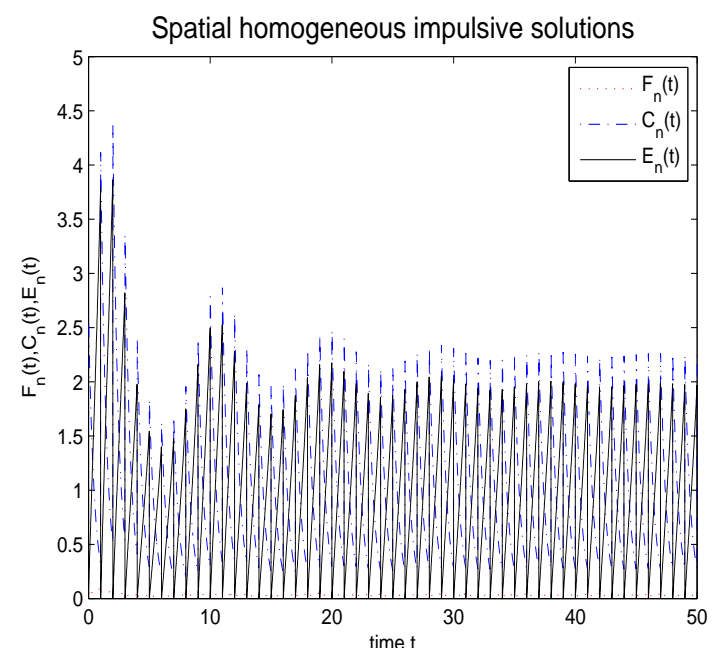

(a) Homogeneous solutions

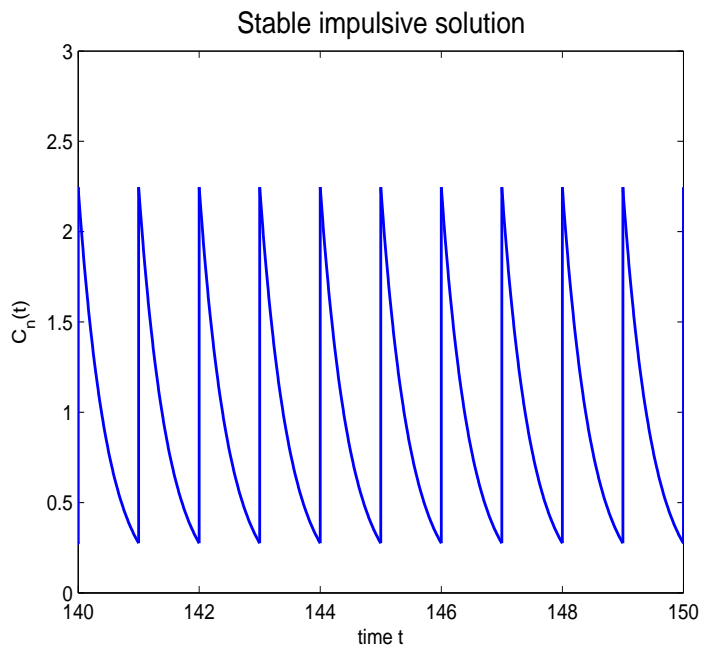

(c) Homogeneous $C_{n}(t)$

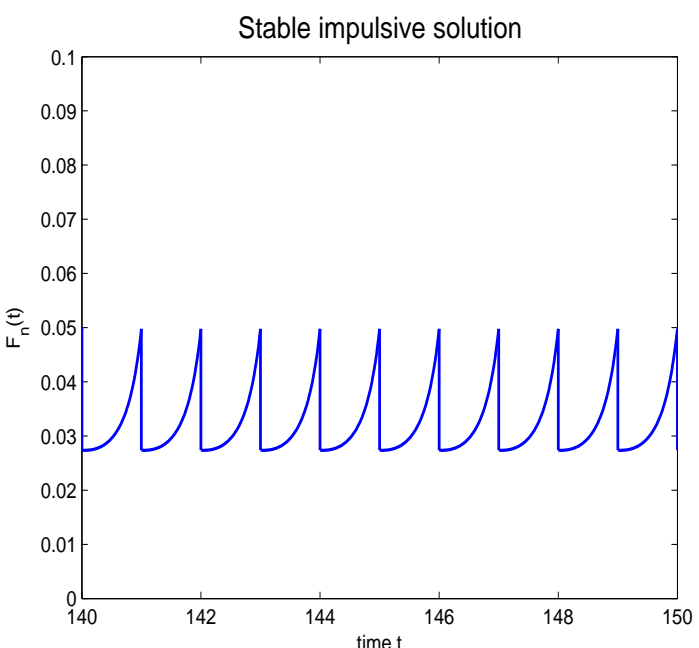

(b) Homogeneous $F_{n}(t)$

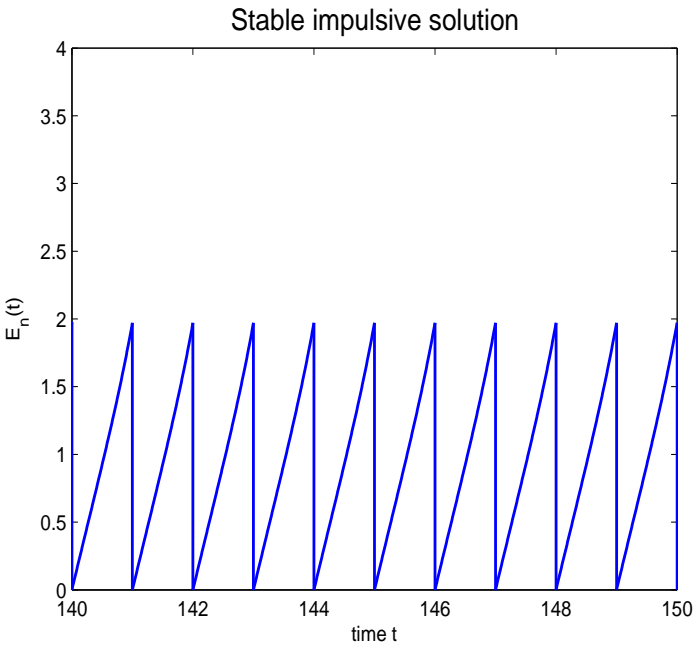

(d) Homogeneous $E_{n}(t)$

Figure 1: Beddington-DeAngelis functional response: stable, spatially homogeneous solutions of (3.57). Panel (a) shows the approach to the impulsive orbit. Panels (b)-(d) show the densities of the three compartments at the impulsive orbit. Parameters are: $\rho=0.55, r_{1}=10, \theta_{1}=0.17$, $\theta_{2}=0.1, m_{1}=2.1, \beta=7, \omega=0.01$, and $\delta_{1}=0.05$. 

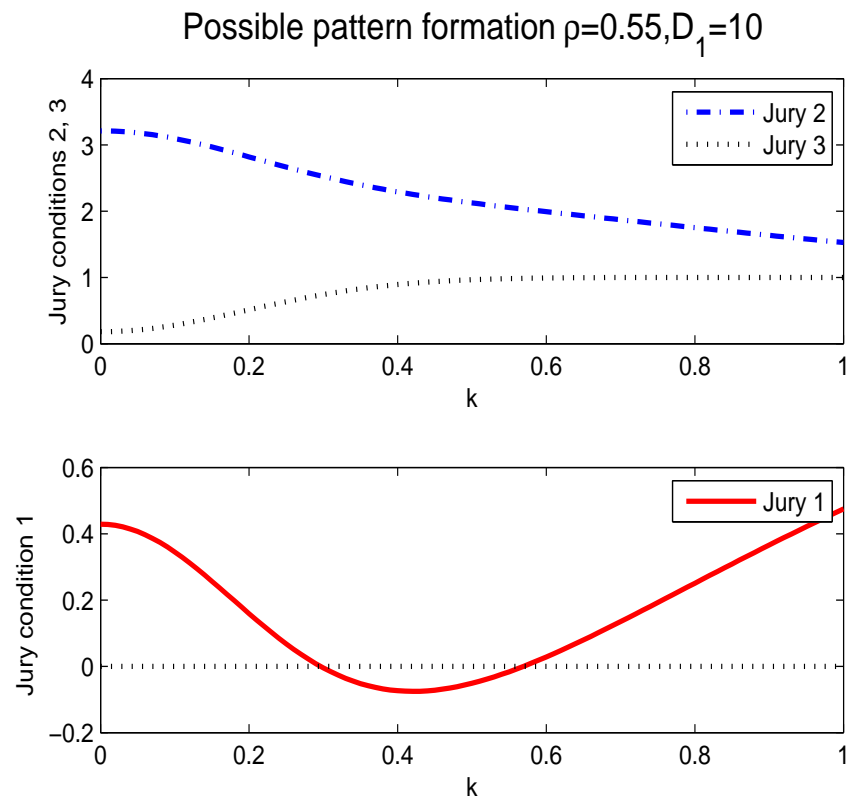

Figure 2: Illustration of conditions in Theorem 3.3 for the Beddington-DeAngelis model (3.57) with impulse conditions (3.4) as a function of spatial mode $k$. The top panel shows that the second and third Jury stability conditions are satisfied. The bottom panel indicates that there is a range of modes where the first condition changes sign. Parameters are as in Figure 1 and $D_{1}=10$.

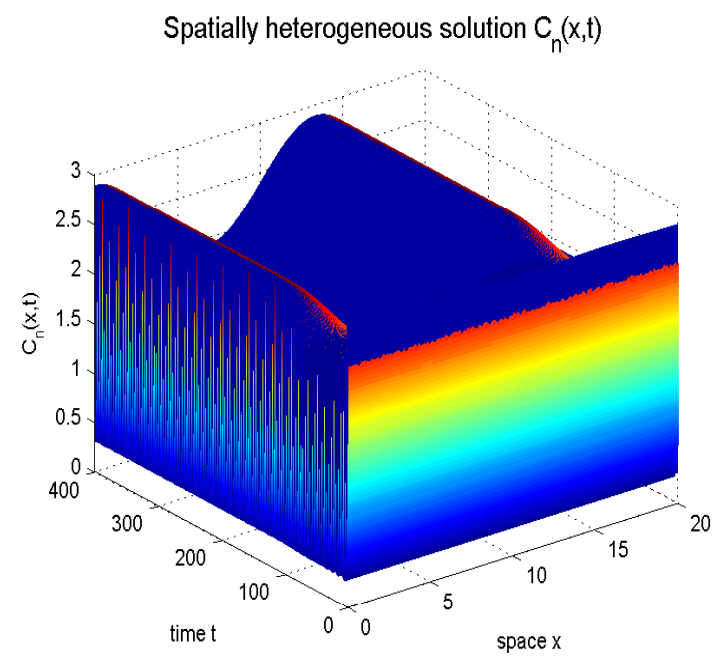

(a) Spatially heterogeneous solution

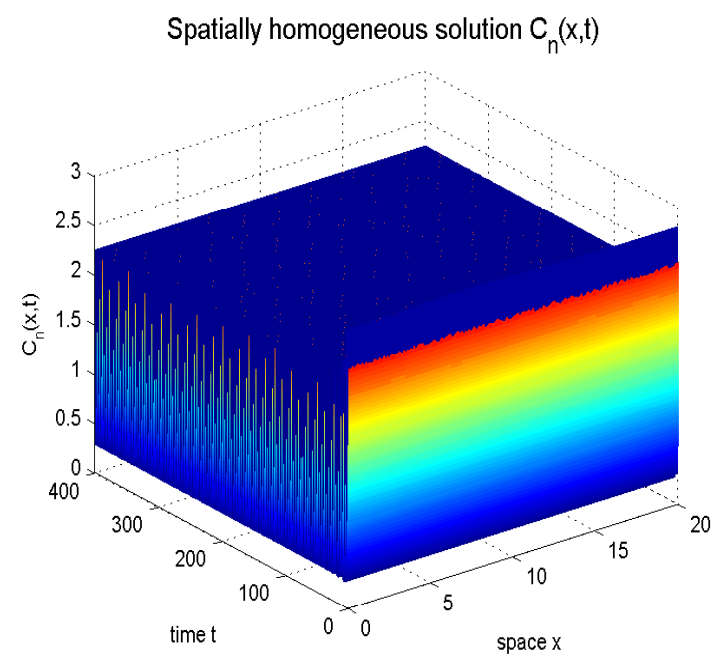

(b) Spatially homogeneous solution

Figure 3: Beddington-DeAngelis functional response: emergence of a spatial pattern with $D_{1}=$ 10 (panel (a)) and convergence to the spatially homogeneous solution with $D_{1}=5$ (panel (b)). Population dynamics parameters are as in Figure 1. Initial conditions are: $F_{n}(0)=0.03+$ $\operatorname{rand}(0,0.01, x), C_{n}(0)=2.4+\operatorname{rand}(0,0.05, x), E_{n}(0)=0.02+\operatorname{rand}(0,0.05, x)$. 


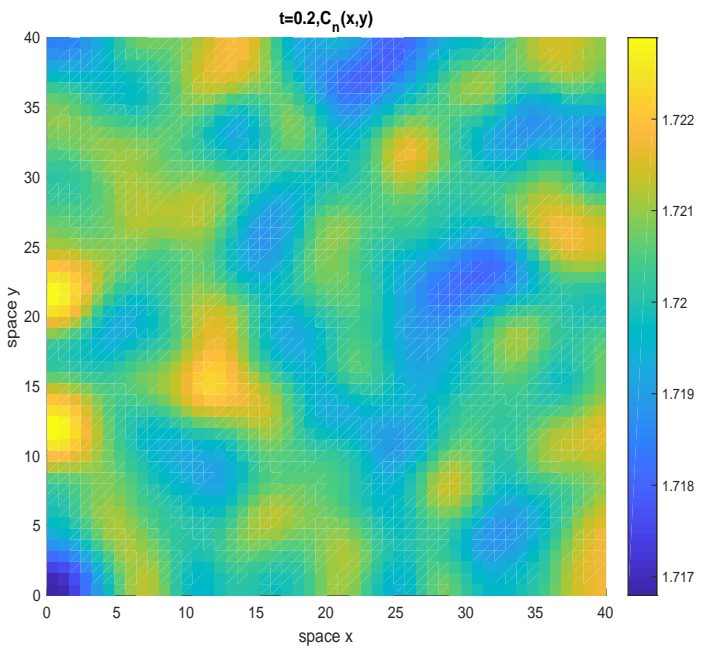

(a) Spatial pattern at $\mathrm{t}=0.2$

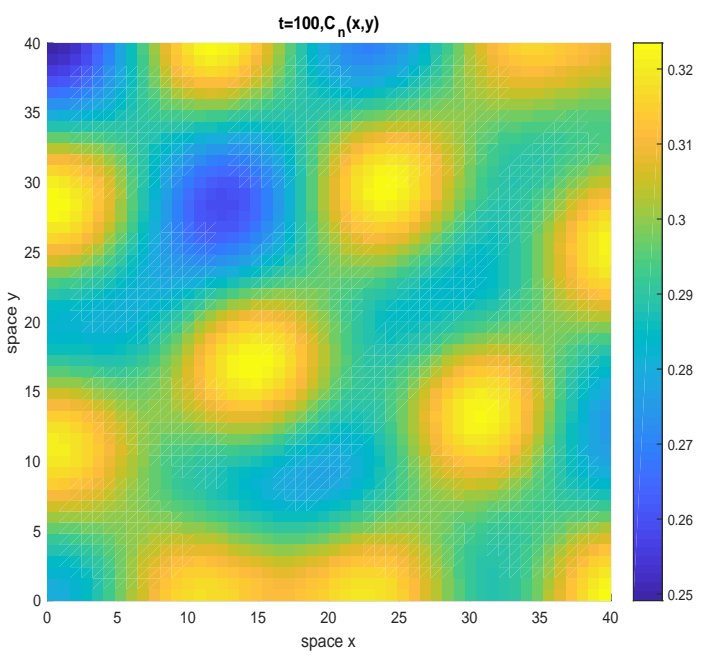

(c) Spatial pattern at $\mathrm{t}=100$

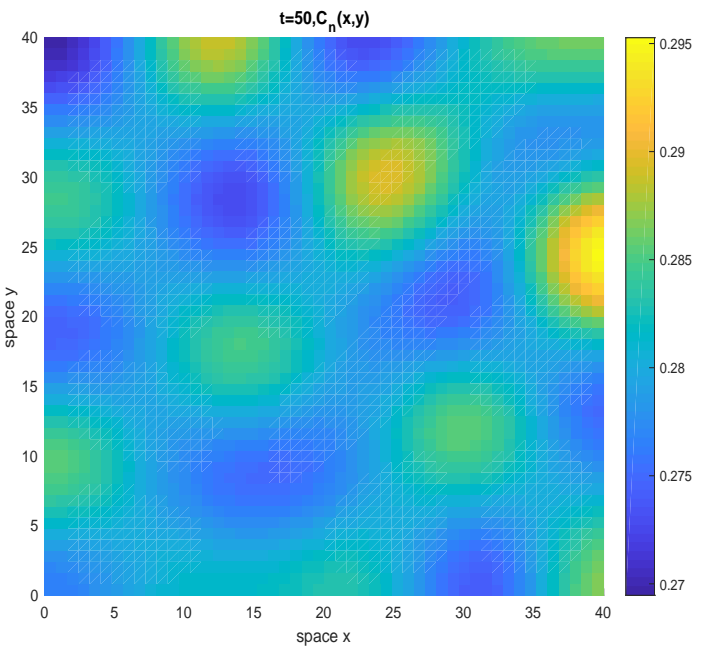

(b) Spatial pattern at $\mathrm{t}=50$

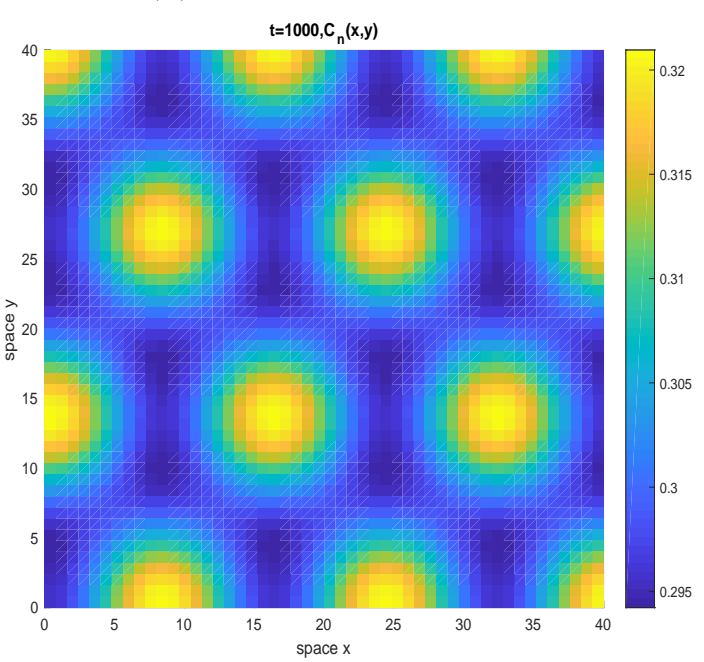

(d) Spatial pattern at $\mathrm{t}=1000$

Figure 4: Beddington-DeAngelis functional response: snap-shots in the emergence of a periodic spot pattern from random initial conditions in a two dimensional region. Only the consumer density is shown. Population dynamics parameters are as in Figure 1 , and $D_{1}=10$. Initial conditions are: $F_{n}(0)=0.04+\operatorname{rand}(0.01,0.05, x, y), C_{n}(0)=2.6+\operatorname{rand}(0.01,0.05, x, y), E_{n}(0)=$ $0.01+\operatorname{rand}(0.01,0.05, x, y)$. 


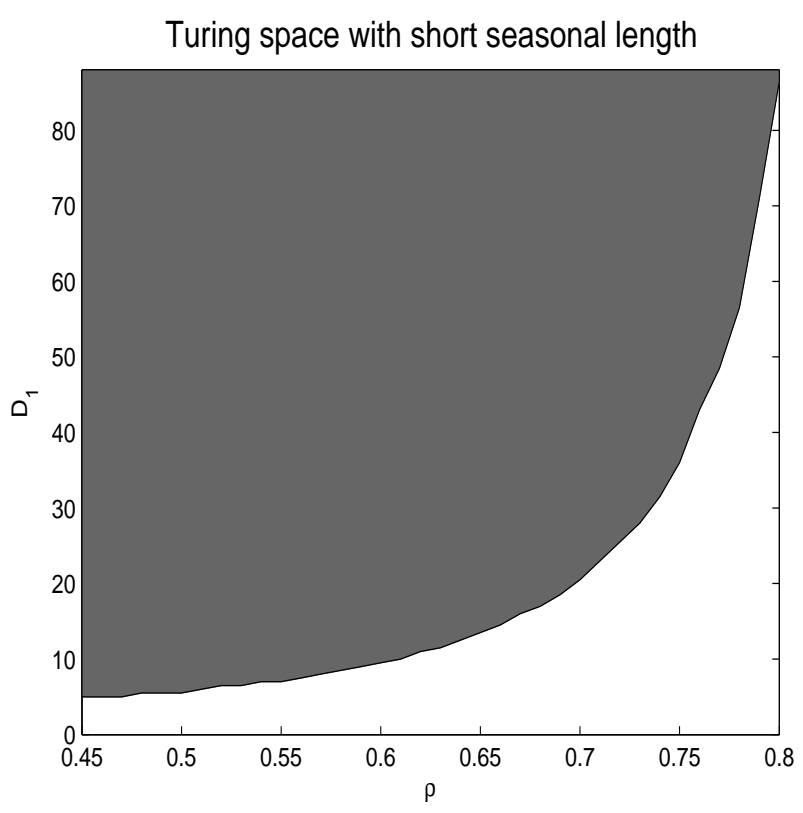

(a) Short seasonal length

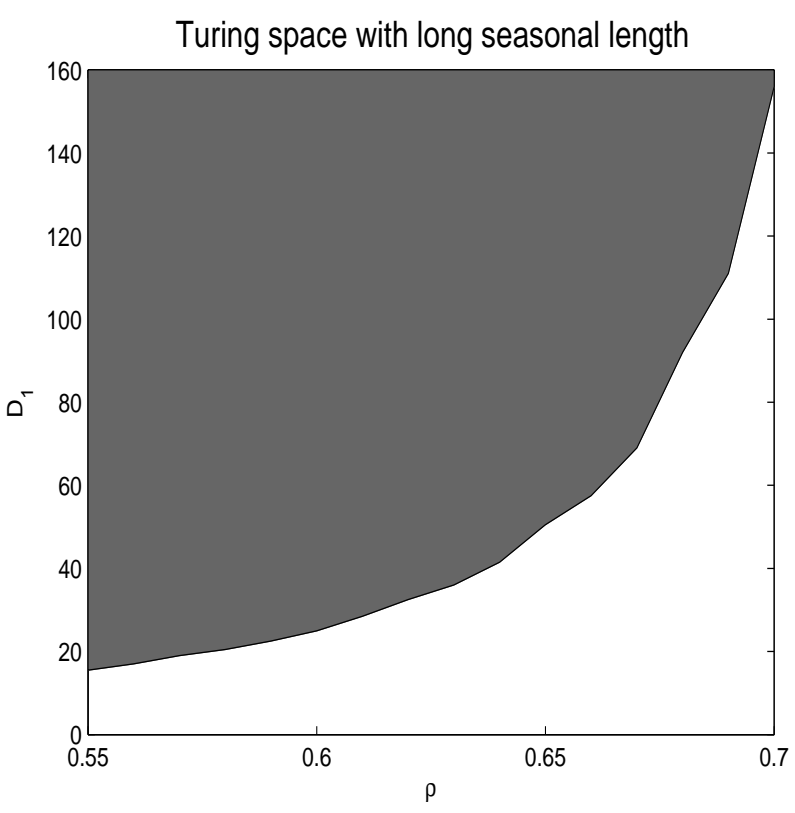

(b) Long seasonal length

Figure 5: Beddington-DeAngelis functional response: parameter space that allows for pattern formation by varying seasonal length $T$. For $5(\mathrm{a})$ and $5(\mathrm{~b})$, we choose $T$ and $1.3 T$ respectively. Parameters are as in Figure 1 unless otherwise noted.

\section{References}

Beddington, J. R. (1975). Mutual interference between parasites or predators and its effect on searching efficiency. Journal of Animal Ecology, pages 331-340.

Benson, D. L., Maini, P. K., and Sherratt, J. A. (1998). Unravelling the Turing bifurcation using spatially varying diffusion coefficients. Journal of Mathematical Biology, 37:381-417.

Bradie, B. (2006). A Friendly Introduction to Numerical Analysis. Pearson Education India.

Cobbold, C. A., Lutscher, F., and Sherratt, J. A. (2015). Diffusion-driven instabilities and emerging spatial patterns in patchy landscapes. Ecological Complexity, 24:69-81.

DeAngelis, D. L., Goldstein, R., and O'Neill, R. (1975). A model for tropic interaction. Ecology, 56:881-892.

Eigentler, L. and Sherratt, J. A. (2018). Analysis of a model for banded vegetation patterns in semi-arid environments with nonlocal dispersal. Journal of Mathematical Biology. https://doi.org/10.1007/s00285-018-1233-y.

Fasani, S. and Rinaldi, S. (2011). Factors promoting or inhibiting Turing instability in spatially extended prey-predator systems. Ecological Modelling, 222:3449-3452. 
Geritz, S. and Gyllenberg, M. (2012). A mechanistic derivation of the DeAngelis-Beddington functional response. Journal of Theoretical Biology, 314:106-108.

Geritz, S. and Kisdi, E. (2004). On the mechanistic underpinning of discrete-time population models with complex dynamics. Journal of Theoretical Biology, 228:261-269.

Holling, C. S. (1959a). The components of predation as revealed by a study of small-mammal predation of the European pine sawfly. The Canadian Entomologist, 91:293-320.

Holling, C. S. (1959b). Some characteristics of simple types of predation and parasitism. The Canadian Entomologist, 91:385-398.

Hsu, S. B., Jiang, J., and Wang, F. B. (2010). On a system of reaction-diffusion equations arising from competition with internal storage in an unstirred chemostat. Journal of Differential Equations, 248:2470-2496.

Huang, Q., Wang, H., and Lewis, M. A. (2017). A hybrid continuous/discrete-time model for invasion dynamics of zebra mussels in rivers. SIAM Journal on Applied Mathematics, 77:854880.

Leslie, P. H. and Gower, J. C. (1960). The properties of a stochastic model for the predator-prey type of interaction between two species. Biometrika, 47:219-234.

Levin, S. A. and Segel, L. A. (1976). Hypothesis for origin of planktonic patchiness. Nature, 259:659.

Lewis, M. A. and Li, B. (2012). Spreading speed, traveling waves, and minimal domain size in impulsive reaction-diffusion models. Bulletin of Mathematical Biology, 74:2383-2402.

Logan, J. D. (2014). Applied Partial Differential Equations. Springer.

May, R. M. (2001). Stability and Complexity in Model Ecosystems, volume 6. Princeton University Press.

Mimura, M. and Murray, J. D. (1978). On a diffusive prey-predator model which exhibits patchiness. Journal of Theoretical Biology, 75:249-262.

Morozov, A., Petrovskii, S., and Li, B. (2006). Spatiotemporal complexity of patchy invasion in a predator-prey system with the Allee effect. Journal of Theoretical Biology, 238:18-35.

Murray, J. D. (2001). Mathematical Biology II. Spatial models and biomedical applications. Springer-Verlag, New York. 
Neubert, M. G., Kot, M., and Lewis, M. A. (1995). Dispersal and pattern formation in a discrete-time predator-prey model. Theoretical Population Biology, 48:7-43.

Pachepsky, E., Nisbet, R. M., and Murdoch, W. W. (2008). Between discrete and continuous: consumer-resource dynamics with synchronized reproduction. Ecology, 89:280-288.

Petrovskii, S. V., Morozov, A. Y., and Venturino, E. (2002). Allee effect makes possible patchy invasion in a predator-prey system. Ecology Letters, 5:345-352.

Rietkerk, M. and Van de Koppel, J. (2008). Regular pattern formation in real ecosystems. Trends in Ecology \& Evolution, 23:169-175.

Sheffer, E., von Hardenberg, J., Yizhaq, H., Shachak, M., and Meron, E. (2013). Emerged or imposed: a theory on the role of physical templates and self-organisation for vegetation patchiness. Ecology Letters, 16:127-139.

Sherratt, J. A. (1995a). Diffusion-driven instability in oscillating environments. European Journal of Applied Mathematics, 6:355-372.

Sherratt, J. A. (1995b). Turing bifurcations with a temporally varying diffusion coefficient. Journal of Mathematical Biology, 33:295-308.

Shi, H. and Ruan, S. (2015). Spatial, temporal and spatiotemporal patterns of diffusive predatorprey models with mutual interference. IMA Journal of Applied Mathematics, 80:1534-1568.

Steele, J. H. (1978). Spatial Pattern in Plankton Communities. Springer Science \& Business Media.

Stocker, T. F., Qin, D., Plattner, G. K., et al. (2014). Climate change 2013: the physical science basis: Working Group I contribution to the Fifth assessment report of the Intergovernmental Panel on Climate Change. Cambridge University Press.

Turing, A. M. (1952). The chemical basis of morphogenesis. Philosophical Transactions of the Royal Society B, 237:37-72.

Vasilyeva, O., Lutscher, F., and Lewis, M. A. (2016). Analysis of spread and persistence for stream insects with winged adult stages. Journal of Mathematical Biology, 72:851-875.

Wang, J., Shi, J., and Wei, J. (2011). Dynamics and pattern formation in a diffusive predatorprey system with strong Allee effect in prey. Journal of Differential Equations, 251:1276-1304. 